\title{
Contact line deposits in an evaporating drop
}

\author{
Robert D. Deegan, Olgica Bakajin, Todd F. Dupont, Greg Huber, Sidney R. Nagel, and Thomas A. Witten \\ James Franck Institute, 5640 South Ellis Avenue, Chicago, Illinois 60637 \\ (Received 14 December 1998; revised manuscript received 26 October 1999)
}

\begin{abstract}
Solids dispersed in a drying drop will migrate to the edge of the drop and form a solid ring. This phenomenon produces ringlike stains and occurs for a wide range of surfaces, solvents, and solutes. Here we show that the migration is caused by an outward flow within the drop that is driven by the loss of solvent by evaporation and geometrical constraint that the drop maintain an equilibrium droplet shape with a fixed boundary. We describe a theory that predicts the flow velocity, the rate of growth of the ring, and the distribution of solute within the drop. These predictions are compared with our experimental results.
\end{abstract}

PACS number(s): 81.15.-z, 68.10.Jy, 47.55.Dz, 83.70.Hq

\section{INTRODUCTION}

The residue left when coffee dries on the countertop or at the bottom of a mug is typically darkest, and hence most concentrated, along the perimeter of the stain giving the deposit a ringlike appearance. Given that the coffee solute was uniformly distributed in the liquid prior to drying, its segregation to the edge is surprising. Ringlike stains are not particular to coffee and are a general phenomenon of which some of the more commonplace examples are mineral rings left on washed glassware, banded deposits of salt on the sidewalk during winter, and enhanced edges in water color paintings (see Fig. 1).

Controlling the distribution of solute during drying is vital in many industrial and scientific processes. For example, paint manufacturers use a variety of additives to ensure that the pigment is evenly dispersed and remains so during drying, and protein crystallographers are attempting to use evaporation driven convection to assemble two-dimensional protein crystals [1,2]. Segregation effects are undesirable in either of these cases. However, as in the production of nanowires [3] or the patterning of a surface [4] the ring effect can be a boon. Understanding the ring formation process should be of interest to those attempting to circumvent or harness this phenomenon.

Ringlike deposits have been previously reported: Bediwi et al. reported on the difficulties of obtaining a uniform deposit [5], Parisse et al. investigated the deformation of sessile drops due to a sol-gel transition of the solute at the boundary [6,7], Adachi et al. studied the stick-slip motion of the contact line of colloidal liquids [8,9], and Conway et al. measured the effect of ring formation on the evaporation rate of sessile drops [10].

With this contribution we expand on an earlier paper [11] in which we reported the experimental results on ring formation and demonstrated that these could be quantitatively accounted for. In our theory, an outward flow in a drying drop of liquid is produced when the contact line is pinned so that liquid that is removed by evaporation from the edge of the drop must be replenished by a flow of liquid from the interior. This flow is capable of transferring $100 \%$ of the solute to the contact line and thus accounts for the strong perimeter concentration of many stains. Furthermore, the theory relies only on a generic property of the substrate-solvent interac- tion, the presence of surface roughness or chemical heterogeneities that produce contact line pinning, and therefore it accounts for the ubiquitous occurrence of ringlike stains.

This paper is arranged in five parts; after this Introduction Sec. II deals with preliminary experiments that show the generality of the phenomenon. Section III lays out the fundamental mechanism responsible for this effect and describes the expected flow mathematically. Section IV presents the calculated results. In Sec. V we present the results of several experiments that test the theory's predictions. We found that most of those predictions are in accord with the experiments. However, there are some discrepancies that suggest the importance of other mechanisms as well.

\section{EXPLORATORY EXPERIMENTS}

Exploratory experiments using a variety of carrier fluids, solutes, and substrates indicated that preferential deposition at the contact line is insensitive to a wide range of experimental conditions. We observed ringlike deposits whenever the surface was partially wet by the fluid irrespective of the chemical composition of the substrate; we tried glass, metal, polyethylene, roughened Teflon, freshly cleaved mica, ceramic, and silicon. Rings were found in big drops $(15 \mathrm{~cm})$ and in small drops $(1 \mathrm{~mm})$. They were found with aqueous and non-aqueous (acetone, methanol, toluene, and ethanol) solvents. They were found with solutes ranging in size from the molecular (sugar and dye molecules) to the colloidal (10- $\mu \mathrm{m}$ polystyrene microspheres) and with solute volume fractions ranging from $10^{-6}$ to $10^{-1}$. Likewise, environmental conditions such as temperature, humidity, and pressure could be extensively varied without affecting the ring.

Effects due to solute diffusion, gravity, electrostatic fields,

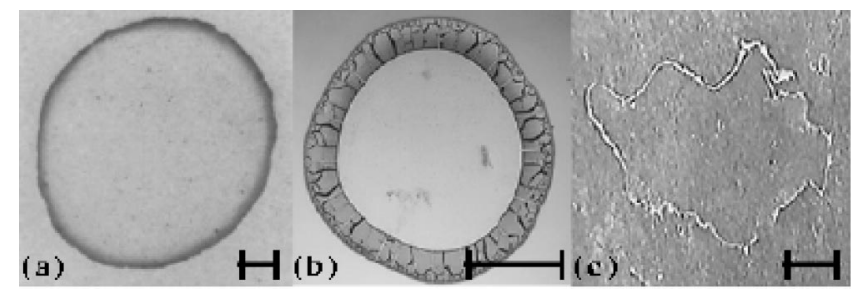

FIG. 1. (a) Coffee stain; (b) dried colloidal microspere; (c) salt deposit. The scale bar corresponds to approximately $1 \mathrm{~cm}$. 
(a)

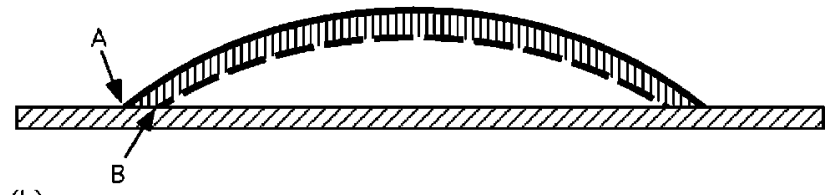

(b)

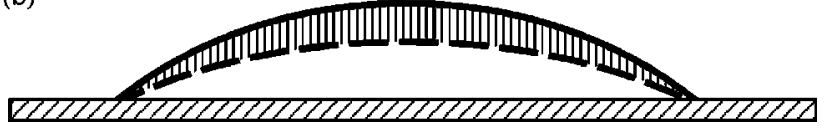

FIG. 2. Schematic illustration of the origin of the advective current. (a) When the contact line is not pinned, uniform evaporation removes the hashed layer, the interface moves from the solid line to the dashed line, and the contact line will move from A to B. However, if the contact line is pinned then the motion from A to B must be prevented by an outflow to replenish the liquid removed from the edge. (b) Shows the actual motion of the interface.

and surface tension forces were considered as possible sources of contact line deposits. We rejected these on the basis of experimental evidence. For these observations we used small drops of a dilute suspension of colloidal particles in water, deposited on a glass microscope slide, and viewed through an optical microscope. We describe the experiment more fully in Sec. V. Diffusion of a solute in the liquid is not the primary agent of segregation because the solute particles clearly move by advection towards the edge of the drop. This can be easily seen under a microscope using large ( $1 \mu \mathrm{m}$ to $10 \mu \mathrm{m})$ colloidal particles. Gravitational effects appear unimportant because the ring produced when the drop is dried upside down (a pendant drop) is indistinguishable from the one produced by a drop dried right side up (a sessile drop.) Likewise, electric fields produced by a charged needle near the contact lines showed no perceptible effect on the ring formation. Several different field configurations with fields of several hundred volts per millimeter were tried without visible effect. Finally, temperature gradients did not seem to play an essential role. When the drop was locally heated with a hot filament, circulating flows appeared, but the ring deposition was not fundamentally changed.

While the effects listed above appear unimportant for ring formation, two other conditions appear necessary: contact line pinning and evaporation from the edge of the drop. When either of these conditions is relaxed the final deposit is uniform. We eliminated pinning by drying the drop on smooth Teflon. The drying drop contracted as it dried and no ring appeared. Likewise we restricted the evaporation (on a glass surface) by covering the drop with a lid that had only a small hole over the center of the drop through which the vapor could escape. This greatly reduced the proportion of evaporation from the perimeter. The resulting deposit was uniform rather than being concentrated at the edge.

\section{THEORY OF SOLUTE TRANSFER}

The essential physical idea behind our theory is that a pinned contact line induces an outward, radial fluid flow when there is evaporation at the edge of the drop. This is graphically demonstrated in Fig. 2. The solid line in Fig. 2(a) represents the initial position of the air-liquid interface. If the evaporation rate is spatially uniform and the contact line is

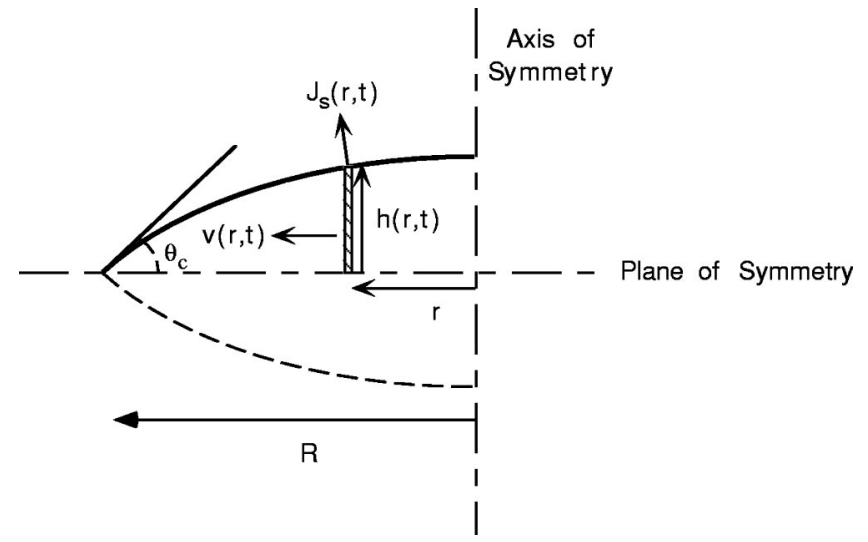

FIG. 3. Schematic of relevant parameters for the theory. The thick solid line represents the air-liquid interface of half a drop viewed from the side. The contact line is defined as the intersection of the interface with the dashed line denoted "plane of symmetry." In an experiment the substrate would define the plane of symmetry. The drop is axisymmetric so that the vertical dashed line denoted the "axis of symmetry" is the axis of rotation. The dashed curve is the reflection of the interface through the plane of symmetry and shows how the boundary-value problem of a drop, detailed in the text, can be replaced by the boundary-value problem of a lens with surfaces defined by the thick solid line and dashed curve line. The diagram also indicates the origin of the divergent evaporation current at the contact line: the discontinuous change in the curvature that occurs as one passes from the upper surface (solid) to the lower surface (dashed).

not pinned, during some interval of time the hashed region would be removed from the drop, the interface would evolve from the solid line to the dashed line, and the contact line would move from A to B. However, if the contact is pinned then there must be a flow that replenishes the liquid that is removed from the edge. In this case of a pinned contact line, the interface evolves as shown in Fig. 2(b) from the solid line to the dashed line and the elasticity of the air-liquid interface, i.e., the tendency of the interface to minimize its surface area, provides the force driving the outward flow of fluid.

We now develop these ideas mathematically starting with the flow inside the drop. We consider an axisymmetric drop as shown in Fig. 3. For the moment we ignore any solute in the liquid. The conservation of fluid determines the relationship between the vertically averaged radial flow of the fluid, $v$, the position of the air-liquid interface, $h$, and the rate of mass loss per unit surface area per unit time from the drop by evaporation, $J_{s}$. The rate of change of the amount of fluid in an infinitesimal annular element at a radial distance $r$ from the center of the drop is equal to the net flux of liquid into the column minus the amount of mass evaporated from the surface of that element:

$$
\rho \frac{\partial h}{\partial t}=-\rho \frac{1}{r} \frac{\partial}{\partial r}(r h v)-J_{s}(r, t) \sqrt{1+\left(\frac{\partial h}{\partial r}\right)^{2}},
$$

where $t$ is time and $\rho$ is the density of the liquid. The effect of $\partial h / \partial r$ term is nearly always small and can be neglected; we keep it here only for completeness. We can solve for $v$ by rewriting this equation in integral form: 


$$
v(r, t)=-\frac{1}{\rho r h} \int_{0}^{r} d r r\left(J_{s}(r, t) \sqrt{1+\left(\frac{\partial h}{\partial r}\right)^{2}}+\rho \frac{\partial h}{\partial t}\right)
$$

from which it is clear that a nonzero $v$ arises when there is a mismatch between the local evaporation rate and the rate of change of the interface.

To compute $v$ we need to specify $h$ and $J_{s}$. The profile of the drop, $h(r, t)$, can be computed from Navier-Stokes equations, however, we will assume, as in Fig. 2, that the shape of the drop is a spherical cap so that the profile is

$$
h(r, t)=\sqrt{\left[\frac{h(0, t)^{2}+R^{2}}{2 h(0, t)}\right]^{2}-r^{2}}-\frac{R^{2}-h(0, t)^{2}}{2 h(0, t)},
$$

where $R$ is the radius of the drop's base and $h(0, t)$ is the height at the center. This corresponds physically to assuming that $R$ is small so that the surface tension, which tends to make drops spherical, is greater than the gravitational force, which tends to flatten drops, and that evaporation occurs slowly so that dynamic contributions to the pressure, such as viscous dissipation, are negligible. In essence, we have neglected all terms in the Navier-Stokes equation, except the pressure term, so that $\nabla p=0$ (i.e., we are in the hydrostatic limit). The time dependence of $h(0, t)$ will be determined below when we compute the evaporation rate.

With $h$ specified we now compute $J_{s}$. The functional form $J_{s}$ depends on whether the rate-limiting step is the transfer rate across the liquid-vapor interface or is the diffusive relaxation of the saturated vapor layer immediately above the drop. In the former case, $J_{s}$ is a constant, while in the later case, as will be shown below, $J_{s}$ is strongly enhanced toward the edge of the drop.

For the case where the limiting rate is the diffusion of the liquid vapor, we assume that the evaporation of the drop rapidly attains a steady state so that the diffusion equation reduces to Laplace's equation

$$
\nabla^{2} u=D \partial_{t} u \simeq 0,
$$

where $u$ is the mass of vapor per unit volume of air and $D$ is the diffusion constant for vapor in air. The assumption is good for times longer than $D / R^{2}$ (e.g., for a 1-mm-sized drop of water this time is $0.04 \mathrm{sec}$ ). The boundary conditions are that (1) along the surface of the drop the air is saturated with vapor so there $u$ is a constant $u_{s}$, (2) the current normal to the substrate $\left(J \cdot n=-D \partial_{n} u\right)$ is zero because the vapor cannot penetrate the substrate, and (3) $u$ converges to the ambient vapor concentration, $u_{\infty}$, far from the drop.

This boundary value problem is identical to that of a charged conductor if we equate $u$ with the electrostatic potential and $J$ with the electric field. As in the case of an electric field near a sharp edge on a conductor (e.g., a lightning rod) the evaporation current diverges near the contact line of the drop (see caption for Fig. 3). Close to the contact line

$$
J_{s}(r, t) \sim(R-r)^{-\lambda},
$$

where $\lambda=\left(\pi-2 \theta_{c}\right) /\left(2 \pi-2 \theta_{c}\right)$ [12] and $\theta_{c}$ is the contact angle the liquid makes with the substrate. This edge enhancement arises from the greater probability of an evapo-
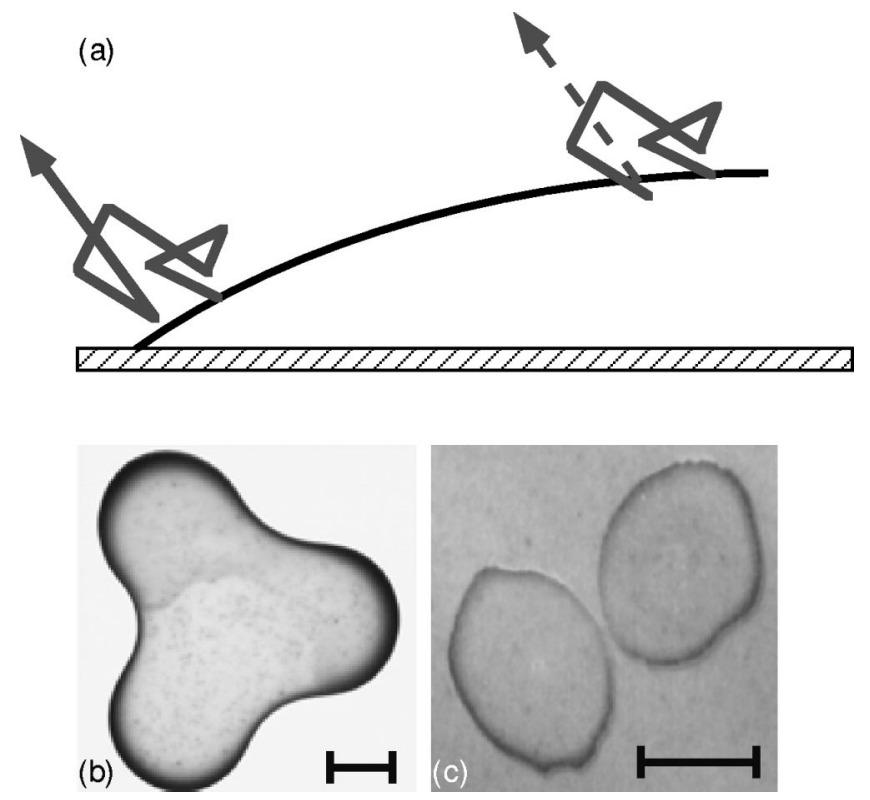

FIG. 4. (a) Shows how the probability of escape of an evaporating molecule is affected by its point of departure. A random walk initiated at the center of the drop results in the molecule being reabsorbed so that the final step is not completed (indicated by the dashed line). However, the same random walk initiated from the edge allows the evaporating molecule to escape. This demonstrates why the evaporation rate is larger at the edge. (b) A clover shaped drop of dried coffee. The extremeties of the drop have the darkest deposits because the probability of escape from these regions is greatest. (c) Two drops dried simultaneously side by side. Region of greatest proximity to each other shows the weakest deposit because, again, the evaporation rate is lowest where the vapor fields of the two drops overlap. The scale bar in (b) and (c) is approximately 1 $\mathrm{cm}$.

rating molecule's escape when leaving from the edge than when leaving from the center of the drop (see Fig. 4). Furthermore, as shown in the Appendix, the expression

$$
J_{s}(r, t) \approx J_{o} f(\lambda)\left[1-(r / R)^{2}\right]^{-\lambda}
$$

is an excellent approximation to the analytic solution to the boundary value problem.

From $J_{s}(r, t)$ we can determine the time dependence of $h(0, t)$. The change of the volume of the drop must be exactly equal to the total evaporation rate. Therefore,

$$
\begin{aligned}
\frac{d M}{d t} & =\rho \frac{d}{d t} \int_{0}^{R} d r^{\prime} 2 \pi r^{\prime} h\left(r^{\prime}, t\right) \\
& =\int_{0}^{R} d r^{\prime} 2 \pi r^{\prime} J_{s}\left(r^{\prime}, t\right) \sqrt{1+\left(\frac{\partial}{\partial r^{\prime}} h\left(r^{\prime}, t\right)\right)^{2}} .
\end{aligned}
$$

While we have assumed steady state, $J_{s}(r, t)$ is nonetheless time dependent because of its dependence on the contact angle, which changes during the course of evaporation. The velocity of the fluid can now be computed by combining Eqs. (2), (4), and (6).

Given the velocity in the drop we can also compute the growth of the ring. We use our knowledge of the initial distribution of solute, namely, that it is constant everywhere in 
the drop at $t=0$, and compute the time it would take a packet of fluid to reach the contact line having started at some initial radial distance $r_{o}$. Within this time all the solute that lay beyond $r_{o}$ becomes part of the ring. Therefore, the mass of solute in the ring $m_{R}(t)$ is

$$
m_{R}\left(t\left(r_{o}\right)\right)=2 \pi c_{o} \int_{r_{o}}^{R} d r^{\prime} r^{\prime} h\left(r^{\prime}, t=0\right),
$$

where $c_{o}$ is the mass of solute per unit volume of solution and $t\left(r_{o}\right)$ is the time it takes to go from $r=r_{o}$ at time $t=0$ to $r=R$ and can be calculated by integration of $d r / d t=v(r, t)$ with the initial condition that $r(0)=r_{0}$. Since we have used the vertically averaged velocity in this formula we have implicitly assumed that there is no vertical segregation of the solute.

Lastly, we can compute the distribution of the solute. However, to do so, without resorting to a lengthier calculation of the flow within the drop, we must assume something about the vertical distribution. For simplicity we start with the assumption, used above, that the concentration, $c(r)$, is a function solely of $r$ (i.e., at a given radial distance the concentration is uniform throughout the liquid column). Therefore, the equation of solute conservation reads

$$
\frac{\partial}{\partial t}(c h)+\frac{1}{r} \frac{\partial}{\partial r}(r c h v)=0,
$$

where $c$ is assumed to be independent of $z$, the vertical distance from the substrate, and the diffusion of solute has been neglected. The mass in the ring can also be determined from the difference of the mass of solute in the drop at $t=0$ and the mass of solute left at a later time, $\int_{0}^{R} d r^{\prime} 2 \pi r^{\prime} h\left(r^{\prime}, t\right) c\left(r^{\prime}, t\right)$.

\section{RESULTS}

In Sec. III we described how to compute the various quantities $\left[v(r, t), m_{R}(t)\right.$, and $\left.c(r, t)\right]$ that describe the state of the drop. Without simplifications, these computations can only be done numerically. However, the qualitative features of the theory can be understood without recourse to numerics. In this section we will highlight these features.

The velocity diverges near the edge of the drop and towards the end of the drying time. These qualitative features can be deduced directly from Eqs. (2) and (5). Near the edge $h \simeq(R-r)$, so that the contribution of the $\partial_{t} h$ term to Eq. (2) is negligible in comparison with the $J_{s}$ term and integration yields $v \sim(R-r)^{-\lambda}$. What is happening physically is that the change of volume of the wedge near the edge (see Fig. 5) becomes increasingly smaller as the contact line is approached and therefore the outgoing vapor must be matched by an equally strong in flow of liquid. In addition, as the height of the wedge decreases the amount of liquid entering the wedge will not vary by much over the drying time because the amount of liquid removed from the surface of the wedge is almost constant. Therefore, $h v_{s}$ must remain constant so that $v_{s} \sim 1 / h$ and diverges as the height goes to zero at the end of the drying.

The ring grows initially as a power law in time. To see this, consider a particle at a distance $R-r$ from the edge. All

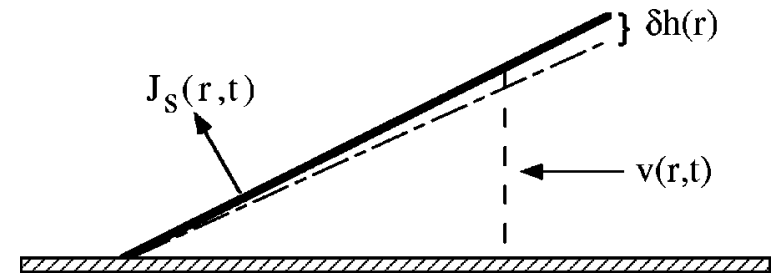

FIG. 5. Illustrates why $v(R-r)$ must scale like $J_{s}(R-r)$. Near the contact line, the compensation for liquid leaving by evaporation through the interface must be increasingly shouldered by the inflow of liquid because the decrease in volume due to lowering the surface becomes increasingly negligible near the contact line.

the particles that are at equal or lesser distance from the contact line will be swept into the ring by the time our particle reaches the edge so that the ring will increase in mass by $m_{R} \sim(R-r)^{2}$. The time this takes is $t \sim \int_{r}^{R} d r^{\prime} / v \sim(R$ $-r)^{\lambda+1}$. Therefore, at early times the ring grows in time as a power law:

$$
m_{R} \sim t^{2 /(1+\lambda)} .
$$

There is also a late time regime in which the growth of the ring rapidly increases and diverges. We can demonstrate this divergence by using a simplified model in which the drop is always thin $h(0, t) \ll R$. In this limit it follows that $\lambda$ is nearly constant because the contact angle does not vary much. For example, for a drop starting with a contact angle of $15^{\circ}, \lambda$ would increase from 0.455 to 0.5 , a $10 \%$ change, over the entire drying time. With $\lambda$ constant it follows from Eq. (7) that the height of the drop decreases linearly in time, $h(0, t)=h(0,0)\left(1-t / t_{f}\right)$, where $h(0,0)$ is the center height at $t=0$ and $t_{f}$ is the total drying time. In this simplified case, the integration of Eq. (8) can be done because the time and space components are separable. This would not be the case for a thick drop where $\lambda$ is time dependent. The result for the thin drop limit is

$$
m_{R}=m_{o}\left[1-\left(1-t / t_{f}\right)^{(1+\lambda) / 2}\right]^{2 /(1+\lambda)}
$$

where $m_{o}$ is the total mass of solute present initially in the drop and $\lambda\left(\theta_{c}\right) \simeq \lambda(0)=1 / 2$. At early times $\left(t \ll t_{f}\right) m_{R}$ $\simeq t^{2 /(1+\lambda)}$ as expected from Eq. (10). At late times $m_{R}-m_{o}$ $\simeq\left(t_{f}-t\right)^{(1+\lambda) / 2}$, which implies that the growth of the ring is divergent: $d m_{R} / d t \simeq 1 /\left(t_{f}-t\right)^{1 / 4}$. It is this final divergence that is responsible for the observed $100 \%$ transfer of the solute to the edge. For a thick drop, where the contact angle varies appreciably with time as the evaporation takes place, the late time result must be obtained numerically. However, the early time results, before the contact angle changes become important, are the same as described above.

\section{EXPERIMENTAL RESULTS}

We studied a dilute colloidal suspension of surfactantfree, charge-stabilized, polystyrene microspheres in water. The mass vs time, shown in Fig. 6(a) for drops of various sizes, is to good approximation linear in time and the evaporation rate rapidly stabilizes to its steady-state value. Thus, the assumption that we can neglect the time dependence in the diffusion equation (4) and that $h(0, t)=h(0,0)\left(1-t / t_{f}\right)$ is supported by data in Fig. 6(a). 

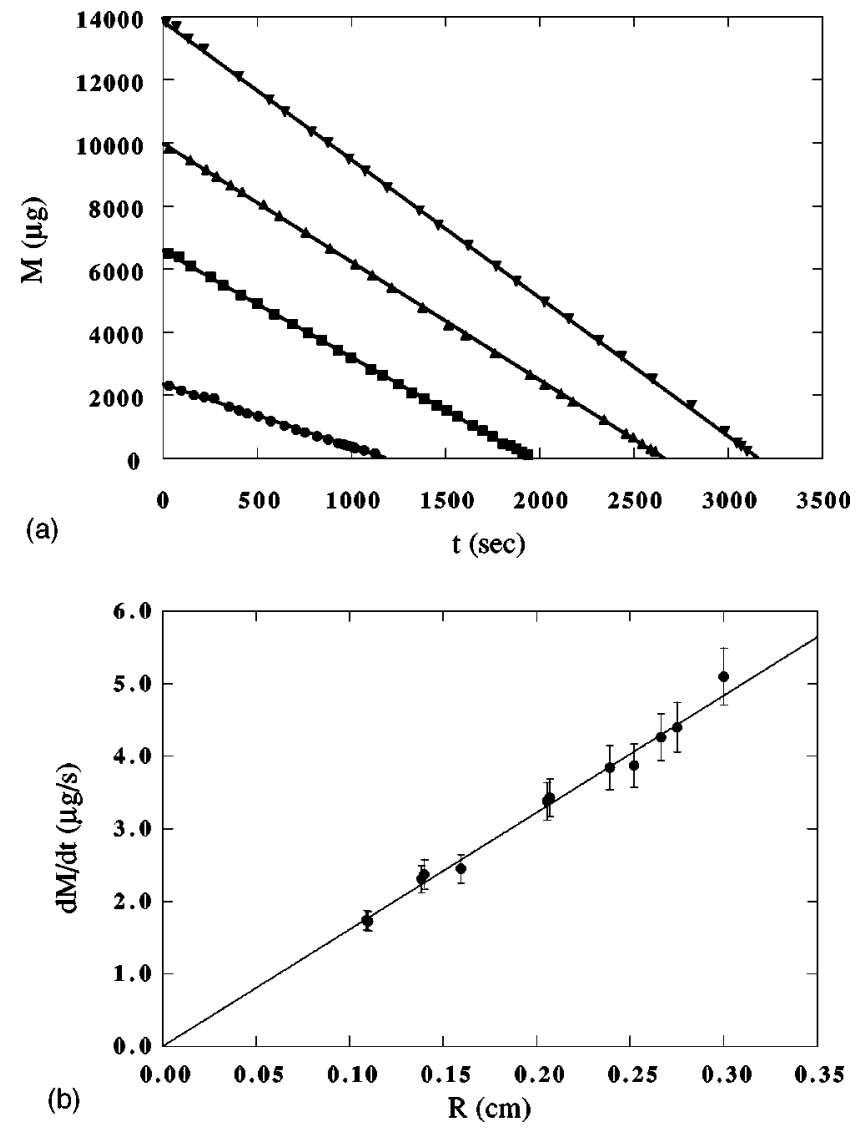

FIG. 6. (a) The mass of the drop $M$ vs time $t$. (b) The rate of mass loss by evaporation, $d M / d t$, normalized to $0 \%$ relative humidity and $20^{\circ} \mathrm{C}$ vs the radius of the drop. The data is well fit by the form $\alpha R$ indicating that the evaporation rate is diffusion limited. The fitting parameter $\alpha=16.1 \pm 0.4 \mathrm{~g} / \mathrm{cm} \mathrm{s}$ is in excellent agreement with the value of $16.5 \mathrm{~g} / \mathrm{cm} \mathrm{s}$, calculated from Eq. (A2).

By extracting the slope of these plots (and others not shown) we can make a plot of evaporation rate vs size of the drop. Intuition might suggest that the evaporation rate should vary as the surface area of the drop $\left(\sim R^{2}\right)$. While this is the case for drops that evaporate uniformly, it is not so for diffusion-limited evaporation. Consider the case of a spherical drop evaporating. The relative humidity around the drop is $u=R / r$ (just like a conductor charged to a potential of 1 ), the local evaporation rate on the surface is $J=-D \partial_{r} u$ $=D R / R^{2}$, and the total evaporation rate is $J \times($ area $)$ $=4 \pi R$, which is linear in $R$. In general, the evaporation rate in the diffusion-limited regime is proportional to $R$ [13]. This counterintuitive result arises because the rate is lessened by the finite probability that an evaporating molecule will return to the drop if it is executing a random walk. Fig. 6(b) demonstrates the rate is linear in $R$ and indicates that the evaporation rate is diffusion limited [14].

Given that the evaporation of our experimental drops is diffusion limited, we can immediately surmise some general qualitative features about the edge deposition process. For an oddly shaped drop, such as the one that resulted in the deposition pattern depicted in Fig. 4(b), the evaporation rate is strongest where there is the lowest density of neighboring evaporation sites. The heavy deposition at tips of the "clover' in Fig. 4(b) arises from the strong evaporation from that location. Also, when two drops are placed next to each other,

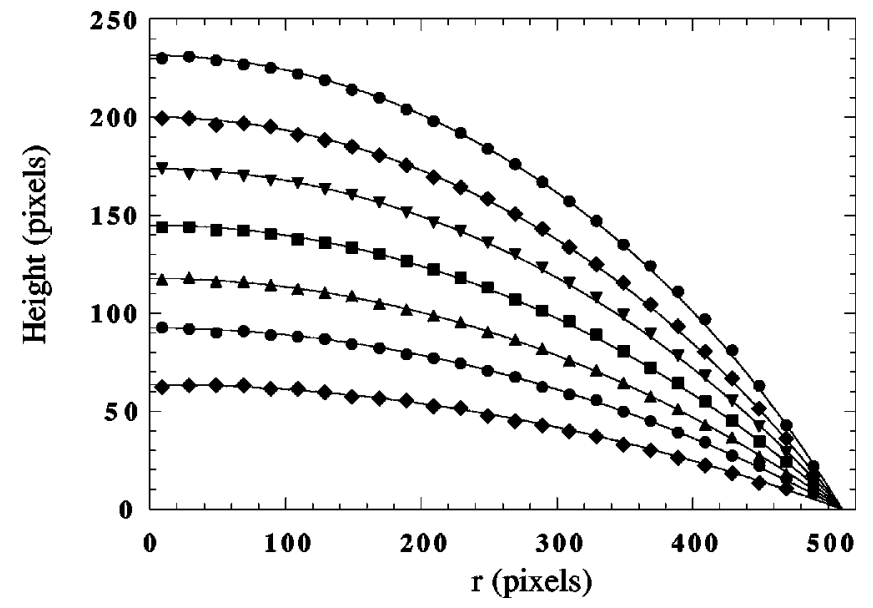

FIG. 7. Plot of the position of the interface above the substrate vs distance from center in units of pixels. The data are taken at equal intervals of time. The solid lines running through the data are fits to a circular profile with the same value of $R$ and $t_{f}$ used for all fits.

their vapor fields overlap and there is less evaporation, hence less deposition, in the region of greatest proximity [see Fig. 4(c)].

By photographing the drop from the side, we measured the shape of the interface at various times. These data are plotted in Fig. 7 for several equally spaced intervals in time. The solid lines running through the data are fits to Eq. (3) using the same parameters $R$ and $t_{f}$ for all fits. Given the good agreement with the data, our assumption of Eq. (3) appears reasonable.

Another feature demonstrated by the data in Fig. 7, is the duration of the pinning. A drop of pure liquid (i.e., without solid components) drying on a substrate is thought to evolve in two distinct modes: an initial pinned phase, in which the base of the drop stays constant and the contact angle decreases, followed by a second unpinned phase, in which the base shrinks but the angle remains fixed $[15,16]$. Our observations of ring-forming drops indicate that the second phase is absent so that the addition of a solid component to the liquid that accumulates at the contact line alters the dynamics of the contact line. In this sense, we believe that the drop is self-pinning: the initial roughness or chemical heterogeneities of the substrate provides a foothold on which the contact line first sticks; the accumulation of material at the contact line, by the process we have been discussing, then apparently strengthens the pinning, and eventually takes over as the primary source of pinning.

The diffusion-limited theory predicts a velocity divergence at the contact line that depends on the contact angle. The velocity in the fluid was measured by tracking the motion of $1-\mu$ microspheres in a drying drop, a small fraction of which were fluorescent. A region extending two-thirds of the way out from the center of the drop up to the contact line was imaged by fluorescent microscopy and recorded on videotape. At roughly $80 \%$ of the drying time the depth of the liquid became comparable to the depth of field of the lens and particles at all depths were in focus. At this stage the contact angle was around $0.2 \mathrm{rad}$, and the corresponding exponent for the velocity was $\lambda=0.47 \pm 0.01$. Several two-sec sequences of images were digitized at $6 \mathrm{~Hz}$ and processed 


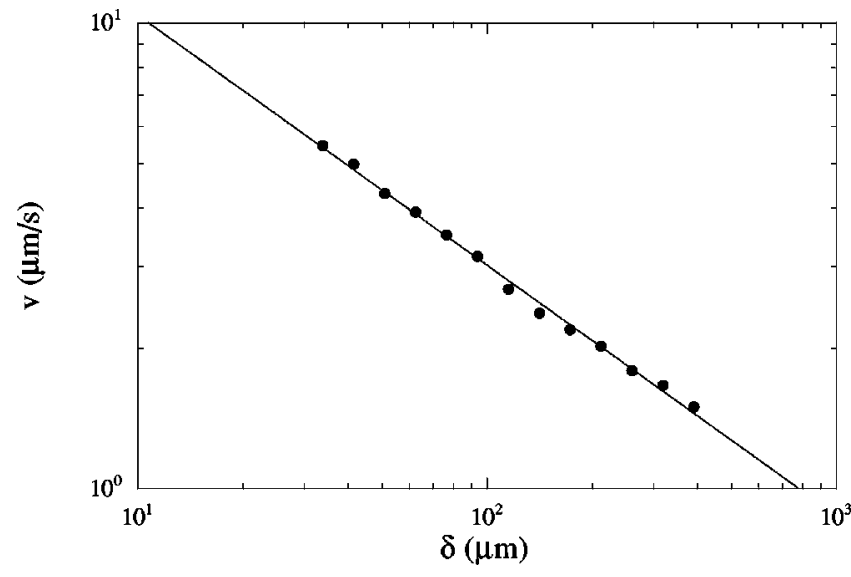

FIG. 8. The $\log$ of the velocity of solute $v$ vs the $\log$ of the distance from the contact line, $R-r$. The solid line is the best fit to a power law and yields an exponent of 0.54 .

using image analysis algorithms for tracking particles [17]. The velocities were binned on a logarithmic scale and plotted vs $\log _{10}(R-r)$ in Fig 8 . We measured an exponent of 0.54 \pm 0.06 (the uncertainty arises from $\pm 5 \mu$ uncertainty in the position of the contact line), which is within error of the predicted value of $0.47 \pm 0.01$.

Several morphological features of the deposition from a drying drop can be appreciated from Eq. (10). Three identical drops were prepared. The first was allowed to evaporate normally. The second was mounted on a pedestal and surrounded by a bath of water such that the level of the bath coincided with the base of the drop. In this case, the evaporation rate is to good spatially uniform approximation. The third drop was surrounded by a chamber with a small hole above the center of the drop so that evaporation primarily occurred at the center. The results of the experiment are shown in Fig. 9. The first and second drop produced ringlike deposits, whereas the third produced a uniform deposit. As is shown schematically to the right of each picture in Fig. 9, the evaporation rates of drops 1 and 2 differ from the profile of the drop and, therefore according to Eq. (2), give rise to a nonzero velocity that is able to rearrange the mass within the drop. However, the third drop had an evaporation profile that was qualitatively similar to the profile of the drop. This produced little redistribution of the solute.

The difference in the thickness of the rings of drop 1 and 2 can also be accounted for qualitatively by our theory. The theory predicts that the first ring will grow at an early time as $t^{2 /(1+\lambda)}$ and that the second ring will grow as $t^{2}$ [because $J_{s}(r, t)=J_{o}$ is constant and therefore $\left.\lambda=0\right]$. Since the two curves must tend towards the same value at the end of the drying (i.e., all the mass must be transferred) the initial growth rate must be larger for the singular evaporation case. The inner edge of the ring can be considered as a solid-liquid interface that moves inward as material arrives. The propagation velocity of the interface is inversely proportional to the height of the liquid surface there since the deposited material piles up to height $h$ at that point (i.e., the solute piles up to the maximum height allowed by the liquid surface). The height is frozen in by the solute accumulated at earlier times; a faster initial growth rate leads to a freezing in of a larger height. Therefore the interface velocity is smaller and thinner rings are grown.
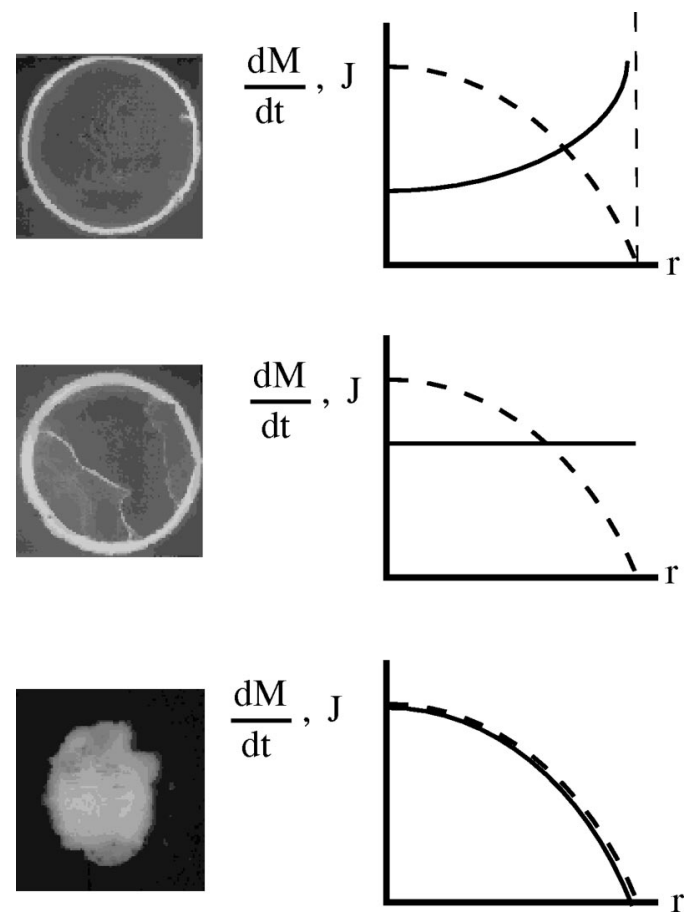

FIG. 9. Photographs of the resulting deposit left by three identical drops dried under different conditions are shown in the left column. The first was allowed to evaporate normally. The second was mounted on a pedestal and surrounded by a bath of water such that the level of the bath coincided with the base of the drop. In this case, the evaporation rate is to good approximation spatially uniform. The third drop was surrounded by a chamber with a small hole above the center of the drop so that evaporation primarily occurred at the center. The radius of the initial drop was approximately $4 \mathrm{~mm}$. The evaporation rate is plotted schematically in the right column. As discussed in the text, the first drop has a singular profile, the second drop has a uniform profile, and the third has a profile that is largest at the center and goes to zero at the edge. The second curve (dashed line) in the right column represents the change of the interface height. When the change of the interface height is different from the evaporation profile, a ring is produced as in the first and second drop. However, if profiles are similar then a uniform deposit is formed. Also note the difference in the ring's thickness in (a) and (b).

Using the same experimental setup as we used to measure the velocity, we counted the total number of microspheres arriving at the contact line at early times. This data is plotted in Fig. 10(a). The solid curve overlaying the data is a oneparameter fit to Eq. (10) with the exponent determined from the measured contact angle. The prefactor to Eq. (10) is

$$
\frac{\ell \phi_{N} \tan \theta_{c}}{2}\left(\frac{(d V / d t)(1+\lambda)}{2^{\lambda} \pi R^{2-\lambda} \tan \theta_{c}}\right)^{2 /(1+\lambda)}
$$

where $\ell$ is the length of the contact line observed, $\phi_{N}$ is the number of microspheres per unit volume, and $d V / d t$ is the evaporation rate of the drop in units of volume per unit time, which can be approximated by $V / t_{f}$, where $V$ is the initial mass of the drop and $t_{f}$ is the total drying time. Despite the excellent fit to the expected power law there is a discrepancy in the value of the prefactor: given the parameters of our experiment, the predicted value of the prefactor is 3.5 but the 

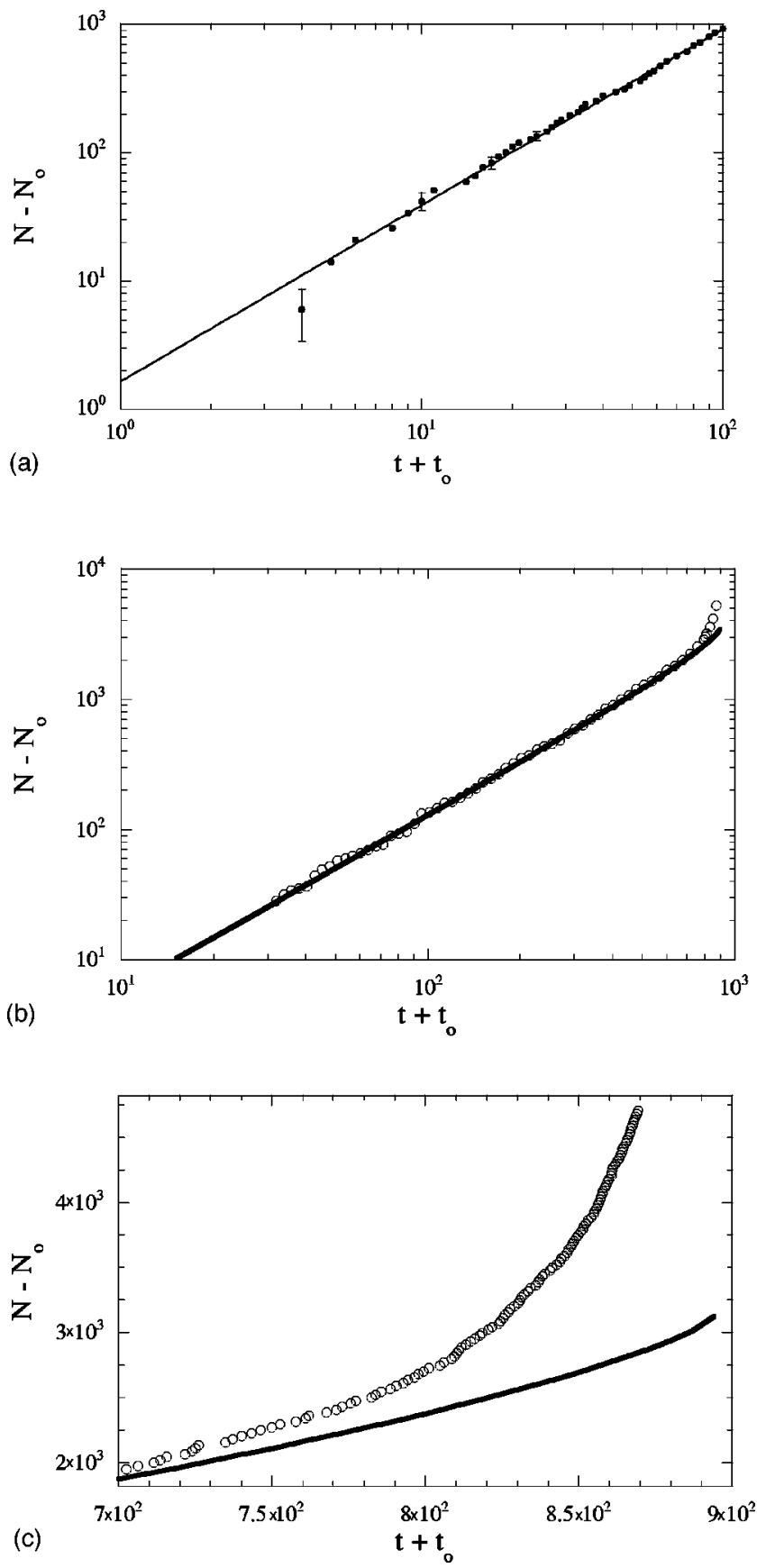

FIG. 10. (a) The number of microspheres $(N)$ arriving at a section of the contact line of length $\ell$ vs time $t$. For this particular run $t_{f}=326 \mathrm{~s}, \phi_{N}=5.4 \times 10^{6} \mathrm{~mm}^{-3}, \ell=21 \mu \mathrm{m}, R=2.08 \mathrm{~mm}$, and $\theta_{c}=0.26 \mathrm{rad}$. The solid line is a fit to $N=N_{p}\left(t / t_{f}\right)^{1.38}$, which yields a value of 1.7 for $N_{p}$. As elaborated on in [11], the $N_{o}$ and $t_{o}$ are adjustments to compensate for the transients as the evaporation rate decays to its steady-state value. For this plot, $N_{o}$ was set to 2 and $t_{0}$ was set to 2. (b) $N$ vs $t$ measured throughout the drying time. (c) Shows a blow up of the late-time behavior (note change to linear scale).

fit in Fig. 10(a) gives a value of 1.7. Therefore, at early times only about $50 \%$ of expected solute is reaching the edge.

Figure 10(b) shows data for the growth of the ring throughout the drying time. Superimposed on the data is a one-parameter fit of the data to a curve obtained by numerical integration of Eq. (11). The theoretical curve is as expected from the above analysis: a steadily increasing growth
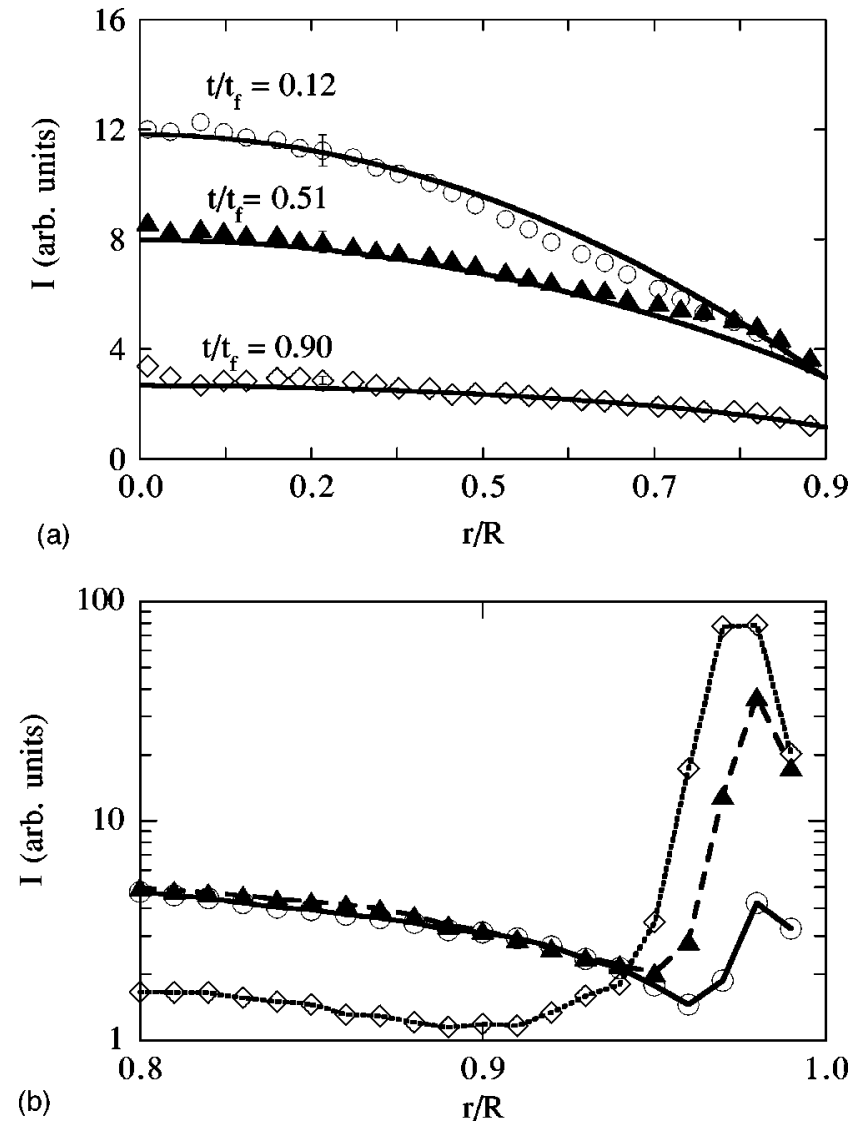

FIG. 11. Intensity of light, $I(r, t)$, for various times from a drop containing flourescent microspheres vs the scaled radius $r / R$. The intensity of light is proportional to $h(r, t) c(r, t)$ and the factor of proportionality is determined by comparing $I(r, 0)$ with $h(r, 0) c(r, 0)$. The solid lines are curves numerically calculated from Eq. (9) and converted into intensity curves using the proportionality factor. The systematic bulge around $\tilde{r} \simeq 0.8$ corresponds to an unanticipated congregation of solute on the surface of the drop. The growth of the ring can be seen in the inset.

rate at early times (power-law behavior) followed by a latetime surge in the growth rate. The fit accounts well for the early time behavior, as it did in Fig. 10(a), however, the theory fails to predict a late-time surge as great as the one seen experimentally. If we force the theory curve to terminate at the same point in the $(N, t)$ plane as the data, then the theory curve will be offset above the data by a multiplicative factor, which for this data set is about 2 . This is in agreement with the $50 \%$ shortfall we found above. To summarize, the experimental data shows and the theory predicts a complete transfer of the solute to the contact line, however, the theory predicts that the material arrives at the contact line earlier than it actually does.

The mass distribution of solute was measured throughout the drying time. A $1-\mu 1$ drop of $0.1-\mu \mathrm{m}$ fluorescent microspheres at $10^{-4}$ volume fraction was placed on a microscope coverslip, isolated from ambient light sources and air currents, and imaged using fluorescent video microscopy. The intensity of fluorescent light, $I(r, t)$, was calibrated and found to be proportional to the number of particles present. A representative set of results for $I(r, t)$ is shown in Fig. 11 for the drop at several stages of evaporation. The solid lines are the profiles for the same reduced time, $t / t_{f}$, calculated by 


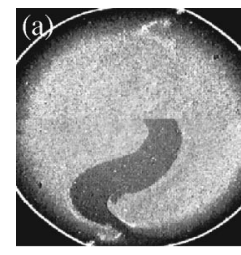

(b)

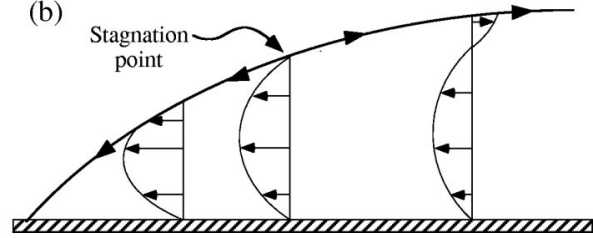

FIG. 12. (a) The surface excess layer of solute revealed by dragging a wire through the drop. The radius of the drop was approximately $2 \mathrm{~mm}$. (b) Circulation pattern in drop due to temperature driven surface flow.

numerical integration of Eq. (9).

Comparison of the calculated curves with the data suggests the presence of an extra solute around $r \simeq 0.8 R$. The effect is more pronounced in drops with larger-sized microspheres where we observe directly a layer of excess material as shown in Fig. 12(a). The layer responds to mechanical probing with a needle as though it were a solid film; it resists shear and it can be fractured. Identifying the surface layer with the excess density in Fig. 11, we estimate from the integrated difference between the observed and theoretical profile that the surface layer accounts for $10 \%$ of the total solute mass in the drop. We observe that the surface cap grows at the expense of the ring as the solute is rerouted back to the center by a inward surface flow. At late times the surface cap breaks up and the particles in it are dragged to the contact line.

\section{DISCUSSION}

Additional effects not accounted for in the theory can modify the deposition significantly. Among these are vertical mixing, viscous stresses, effects of solute interaction, diffusion, sedimentation, and Marangoni effects. A homogeneous vertical distribution of solute was assumed for Eq. (9), which is equivalent to assuming that vertical mixing is vigorous. It is unlikely that this is physically realized, so that the theory would need to be modified to include stratification effects. Viscous stresses may in principle perturb the drop away from the spherical-cap shape we assumed. As the velocity diverges near the edge so does the viscous term in NavierStokes equation $\left(\nabla^{2} v \sim \partial_{z}^{2} v \sim v / h^{2}\right)$ so that at some finite distance from the edge the surface of the drop will be distorted. For a low viscosity liquids, such as water, this length scale is insignificant. However, for higher viscosity liquids it may become a significant fraction of the radius. For a more concentrated solution the viscosity of the fluid is altered and the physical dimensions of the ring may be large. Both of these effects may distort the shape of the drop. If the size of the solute particles is small, diffusive currents become comparable to the advective currents and Eq. (9) would need to be modified. Finally, if the size of the solute particles becomes too large, sedimentation of the solute will affect the amount of material arriving at the edge. This may account for some of the shortfall discussed earlier. These effects provide additional avenues to be explored in surface deposition processes.

We believe that Marangoni flows are a significant factor in our experiments. For a water drop drying on a glass substrate the apex of the drop ought to be coolest, despite it being the point of least evaporative heat loss, because it is furthest from the substrate, which is the drop's primary heat source. Surface tension decreases with rising temperature, and therefore the surface tension would be greatest at the pole of the drop. This gradient would drive a radially inward surface flow. However, it is difficult to obtain a quantitative prediction of the flow speed because there are a number of additional effects of unknown strength—such as surface pressure due to an adsorbed surfactant or solute, and surface viscosity in the presence of adsorbed molecules - that tend to counteract Marangoni flows.

Nonetheless, the phenomena we observe in the drop are qualitatively in agreement with what is expected given the temperature distribution. We expect and observe an inward flow. We also expect that there will be a stagnation point beyond which the surface flow will be outward. This reversal of direction would occur at the radial point at which the drag on the surface from an underlying liquid, which is diverging with the velocity as the contact line is approached, overcomes the oppositely directed surface tension gradient [see Fig. 12(b)]. We observe such a stagnation point. Furthermore, as the drop dries we expect that this point will move towards the center of the drop because the overall scale of the bulk flow velocity, and hence the drag on the surface, increases as the height of the drop decreases. The ultimate dispersal of the surface cap observed experimentally is consistent with this scenario.

Given the 50\% shortfall measured for the ring growth, it is appropriate to question the success of our theory. The pragmatic answer is that the theory is an increasingly useful, predictive tool in the limit of small solute particles. The ring growth measurement was done with $1-\mu \mathrm{m}$ particles and the theory could account for 50\% of the observed behavior. For the concentration measurement $0.1-\mu \mathrm{m}$ particles were used and accuracy of the theory improved significantly. For a solute of molecular size there is no discernible stratification as is seen in drops of $1-\mu \mathrm{m}$-sized microspheres (Fig. 12). Another answer is that our theory captures the essential mechanism for ring formation and that the understanding achieved through it is not diminished by the presence of other types of flows that ultimately do not alter the fact that the $100 \%$ of the solute is transferred to the edge.

\section{CONCLUSION}

Despite its shortcomings, our theory is a useful tool for understanding and predicting the ring-formation process. It accounts for the widespread occurrence of solute rings, because few ingredients are required-a weakly pinning substrate and evaporation-and these ingredients occur commonly; it accounts in a natural way for nearly complete transfer of the solute to the periphery; and it indicates how manipulating the vapor field around the drop provides a means of controlling the deposition process.

The potential for controlling deposition on a surface using this contact line deposition remains virtually unexplored. Two aspects seem particularly promising. On the applied side, this mechanism provides a means of concentrating a deposit into fine lines, which may rival or exceed what is currently possible using lithographic or etching processes. Such lines can be useful for exploring electronic quantum 
confinement or for writing information at high density. Achieving narrow deposits requires increasing dilution of the depositing solute. Thus we are led to explore contact-line deposition when the solute becomes more and more dilute. On the fundamental side, our mechanism seems to rely on a mutual reinforcement between contact-line pinning and deposition. This reinforcement appears crucial to the ringforming process, but has yet to be explored. This reinforcement must weaken, and ultimately fail, as the solute is diluted. The mechanism of this failure would seem to have widespread implication of its own. Here too, the regime of interest is the regime of highly dilute solutes. Our studies of this regime are in progress.

\section{ACKNOWLEDGMENTS}

We thank H. Li, X. Shi, and M. Baildon for their early contributions to this project; J. Crocker, D. Grier, and A. Marcus for sharing their expertise, their image analysis code, and their facilities; and S. Garoff, L. Mahadevan, S. Esipov, R. Leheny, D. Mueth, E. Ehrichs, J. Knight, S. Blanton, N. Menon, J. Cina, L. Kadanoff, T. Halsey, and H. Zhou for valuable discussions and suggestions. This work was supported by NSF Grant No. DMR-9410478 and the MRSEC Program of the NSF under Grant No. NSF DMR-9808595.

\section{APPENDIX: THE SOLUTION TO THE BOUNDARY VALUE PROBLEM OF A LENS-SHAPED CONDUCTOR}

The problem of a spherical cap on an impermeable substrate can be replaced by the problem of a lens (as shown in Fig. 3) in free space. The general solution for the electrostatic potential of a charged conductor with a shape defined by two intersecting spheres is given in Ref. [18]. This solution for our specific problem (translated into the language of diffusion) is

$$
\begin{aligned}
u(\alpha, \beta)= & u_{\infty}+\left(u_{s}-u_{\infty}\right) \sqrt{2(\cosh \alpha-\cos \beta)} \\
& \times \int_{0}^{\infty} d \tau P_{-1 / 2+\imath \tau}(\cosh \alpha) \\
& \times \frac{\cosh \theta_{c} \tau \cosh (2 \pi-\beta) \tau}{\cosh \pi \tau \cosh \left(\pi-\theta_{c}\right) \tau},
\end{aligned}
$$

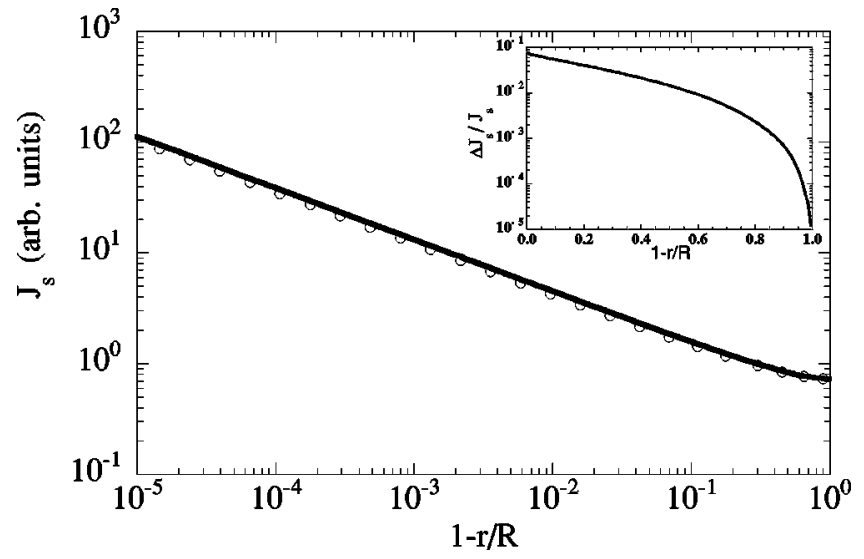

FIG. 13. The value of $J_{s}$ calculated with the full analytic form compared to the approximate form. The two curves are approximately equal. As is shown in the inset, the upper bound on the difference is $10 \%$.

where $P_{-1 / 2+\imath \tau}(x)$ are Legendre functions of the first kind, $\alpha$ and $\beta$ are toroidal coordinates in which toroidal surfaces are labeled with $\alpha$ and spherical surfaces with $\beta$, and the two surfaces are at $\beta_{1}=3 \pi-\theta_{c}$ and $\beta_{2}=\pi+\theta_{c}$. Since we are interested in the evaporation at the surface we calculate $J$ $=-D \nabla u$ normal to the surface:

$$
\begin{aligned}
J_{s}= & \left.D(\cosh \alpha+\cos \beta) \partial_{\beta} u\right|_{\beta=3 \pi-\theta_{c}} \\
= & -D\left(u_{s}-u_{\infty}\right)\left[\sin \left(\theta_{c}\right) / 2+\sqrt{2}(x\right. \\
& \left.+\cos \theta_{c}\right)^{3 / 2} \int_{0}^{\infty} d \tau P_{-1 / 2+}(x) \tau \\
& \left.\times \frac{\cosh \theta_{c} \tau \tanh \left(\pi-\theta_{c}\right) \tau}{\cosh \pi \tau}\right],
\end{aligned}
$$

where $r=R \sqrt{1-x^{2}} /\left(x+\cos \theta_{c}\right)$ where $R$ is the radius of the drop and $x$ is a parameter ranging from 0 to 1 .

The value of Eq. (A2) was computed numerically and compared to the approximate form given in Eq. (6). In Fig. 13 both $J$ exact and $J$ approximate are plotted. The inset is a plot of the percent difference between the two $J_{\mathrm{s}}$; as can be seen the error is no greater than $10 \%$.
[1] N. D. Denkov, O. D. Velev, P. A. Kralchevsky, I. B. Ivanov, H. Yoshimura, and K. Nagayama, Langmuir 8, 3183 (1992).

[2] A. S. Dimitrov, C. D. Dushkin, H. Yoshimura, and K. Nagayama, Langmuir 10, 432 (1994).

[3] T. Ondarçuhu and C. Joachim, Europhys. Lett. 42, 215 (1998).

[4] J. Boneberg, F. Burmeister, C. Shäfle, P. Leiderer, D. Reim, A. Fery, and S. Herminghaus, Langmuir 13, 7080 (1997).

[5] A. B. El Bediwi, W. J. Kulnis, Y. Luo, D. Woodland, and W. N. Unertl, Mater. Res. Soc. Symp. Proc. 372, 277 (1995).

[6] F. Parisse and C. Allain, J. Phys. II 6, 1111 (1996).

[7] F. Parisse and C. Allain, Langmuir 13, 3598 (1996).

[8] E. Adachi, A. S. Dimitro, and K. Nagayama, in Film Formation in Waterborne Coatings, edited by T. Provder, M. A.
Winnik, and M. W. Urban (American Chemical Society, Washington, D.C., 1996), p. 419.

[9] E. Adachi, A. S. Dimitro, and K. Nagayama, Langmuir 11, 1057 (1995).

[10] J. Conway, H. Korns, and M. R. Fisch, Langmuir 13, 426 (1997).

[11] R. D. Deegan, O. Bakajin, T. F. Dupont, G. Huber, S. R. Nagel, and T. A. Witten, Nature (London) 389, 827 (1997).

[12] J. D. Jackson, Classical Electrodynamics 1st ed. (Wiley, New York, 1962).

[13] J. T. Davies and E. K. Rideal, Interfacial Phenomenon (Academic Press, New York, 1963).

[14] This is a well-known result. For example, see K. Histek et al., 
J. Appl. Phys. 73, 7395 (1993).

[15] C. Bourgès-Monnier and M. E. R. Shanahan, Langmuir 11, 2820 (1995).

[16] M. E. R. Shanahan, Langmuir 11, 1041 (1995).
[17] J. C. Crocker and D. G. Grier, J. Colloid Interface Sci. 179, 298 (1996).

[18] N. N. Lebedev, Special Functions and Their Applications (Prentice-Hall, Englewood Cliffs, NJ, 1965). 